Louisiana State University

LSU Digital Commons

Faculty Publications

Department of Physics \& Astronomy

$12-1-2007$

\title{
Monitoring the low-energy gamma-ray sky using Earth occultation with GLAST GBM
}

\author{
G. L. Case \\ Louisiana State University \\ C. A. Wilson-Hodge \\ NASA Marshall Space Flight Center \\ M. L. Cherry \\ Louisiana State University \\ R. M. Kippen \\ Los Alamos National Laboratory \\ J. C. Ling \\ Jet Propulsion Laboratory
}

See next page for additional authors

Follow this and additional works at: https://digitalcommons.Isu.edu/physics_astronomy_pubs

\section{Recommended Citation}

Case, G., Wilson-Hodge, C., Cherry, M., Kippen, R., Ling, J., Radocinski, R., \& Wheaton, W. (2007). Monitoring the low-energy gamma-ray sky using Earth occultation with GLAST GBM. AIP Conference Proceedings, 921, 538-539. https://doi.org/10.1063/1.2757440

This Conference Proceeding is brought to you for free and open access by the Department of Physics \& Astronomy at LSU Digital Commons. It has been accepted for inclusion in Faculty Publications by an authorized administrator of LSU Digital Commons. For more information, please contact ir@lsu.edu. 


\section{Authors}

G. L. Case, C. A. Wilson-Hodge, M. L. Cherry, R. M. Kippen, J. C. Ling, R. G. Radocinski, and W. A. Wheaton 


\title{
Monitoring the Low-Energy Gamma-Ray Sky Using Earth Occultation with GLAST GBM
}

\author{
G. L. Case ${ }^{*, \dagger}$, C. A. Wilson-Hodge ${ }^{* *}$, M. L. Cherry*, R. M. Kippen ${ }^{\star}$, J. C. Ling ${ }^{\S}$, R. \\ G. Radocinski ${ }^{\S}$ and W. A. Wheaton \\ ${ }^{*}$ Louisiana State University, Baton Rouge, LA 70803 \\ $\dagger$ Southern University, Baton Rouge, LA 70813 \\ ${ }^{* *}$ Marshall Space Flight Center, Huntsville, AL 35812 \\ Los Alamos National Laboratory, Los Alamos, NM 87545 \\ §et Propulsion Laboratory, Caltech, Pasadena, CA 91109 \\ IIfrared Processing and Analysis Center, Caltech, Pasadena, CA 91125
}

\begin{abstract}
Long term all-sky monitoring of the $20 \mathrm{keV}-2 \mathrm{MeV}$ gamma-ray sky using the Earth occultation technique was demonstrated by the BATSE instrument on the Compton Gamma Ray Observatory. The principles and techniques used for the development of an end-to-end earth occultation data analysis system for BATSE can be extended to the GLAST Burst Monitor (GBM), resulting in multiband light curves and time-resolved spectra in the energy range $8 \mathrm{keV}$ to above $1 \mathrm{MeV}$ for known gamma-ray sources and transient outbursts, as well as the discovery of new sources of gamma-ray emission. In this paper we describe the application of the technique to the GBM. We also present the expected sensitivity for the GBM.
\end{abstract}

Keywords: GLAST Burst Monitor; gamma-ray observations; Earth occultation

PACS: 95.10.Gi; 95.55.Ka; 95.80.+ + ; 95.85.Pw

The hard $x$-ray/low-energy gamma-ray energy region $(>20 \mathrm{keV})$ is becoming an increasingly important window into the high-energy processes occurring in a variety of astrophysical objects, including $\mathrm{x}$-ray binaries, AGN, anomalous $\mathrm{x}$-ray pulsars (AXPs), etc. Many of these objects undergo transient episodes, and long term, high duty cycle monitoring of the temporal and spectral characteristics of these sources, particularly during transient events, is essential to better understanding the physical processes that drive the observed emission.

The Burst and Transient Source Experiment (BATSE) instrument aboard the Compton Gamma-ray Observatory (CGRO) performed long-term (April 1991 - June 2000) monitoring of the gamma-ray sky in the energy range from $\sim 20 \mathrm{keV}$ to $1.8 \mathrm{MeV}$ using the Earth occultation technique. The Earth occultation technique involves looking for "steps" in the count rate of a detector both before and after an astronomical source is occulted by the Earth. After subtracting off the detector background, the difference in the count rates before and after occultation gives the count rate due to the occulted source. For the CGRO orbit, $88 \%$ of the sky underwent Earth occultation each day, and $100 \%$ of the sky was observed every $\sim 50$ days with a duty cycle of $>50 \%$. The algorithms and analysis software were developed and implemented that enabled the production of light curves with 1-day resolution and spectra from $\sim 20$ $\mathrm{keV}$ to $1.8 \mathrm{MeV}[1,2]$. Using the occultation technique, BATSE monitored known transient objects, such as Galactic black hole candidates (BHCs), neutron star binaries, and AGN. Many of these objects had been previously detected by other missions (e.g. RXTE, BeppoSAX, Granat, Ginga, etc.), though some were actually discovered by BATSE. The database compiled using the BATSE occultation method $[1,3]$ has proven to be a rich source of information for the study of these transient objects, as well as persistent sources such as neutron star binaries, AGN, pulsars, etc. [4, 5, 6].

Currently, there are no instruments in orbit that can provide nearly continuous all-sky monitoring of the low-energy gamma-ray sky. The launch of GLAST will provide an opportunity to perform this monitoring using the GLAST Burst Monitor (GBM) detectors and the Earth occultation technique. The GBM consist of $12 \mathrm{NaI}$ detectors, $12.7 \mathrm{~cm}$ in diameter and $1.27 \mathrm{~cm}$ thick, covering the energy range from $8-1000 \mathrm{keV}$ [7]. Six of these detectors are oriented perpendicular to the z-axis of GLAST and $60^{\circ}$ apart in azimuth. These six detectors have a good view of the Earth's limb. Four detectors are oriented $45^{\circ}$ from the spacecraft zenith and have a limited view of the limb. The remaining two detectors will not have the Earth's limb in their field of view. The GBM has $2 \mathrm{BGO}$ detectors, $12.7 \mathrm{~cm}$ in diameter and $12.7 \mathrm{~cm}$ thick, covering the energy range from $0.150-25 \mathrm{MeV}$. These two detectors are located on opposite sides of the spacecraft and have a full view of the Earth's limb. Together, the NaI and BGO detectors will provide continuous monitoring of the majority of the gamma-ray sky from $\sim 8 \mathrm{keV}$ up to $>1 \mathrm{MeV}$. The occultation software and analysis

CP921, The First GLAST Symposium

edited by S. Ritz, P. Michelson, and C. Meegan

(C) 2007 American Institute of Physics 978-0-7354-0431-1/07/\$23.00 


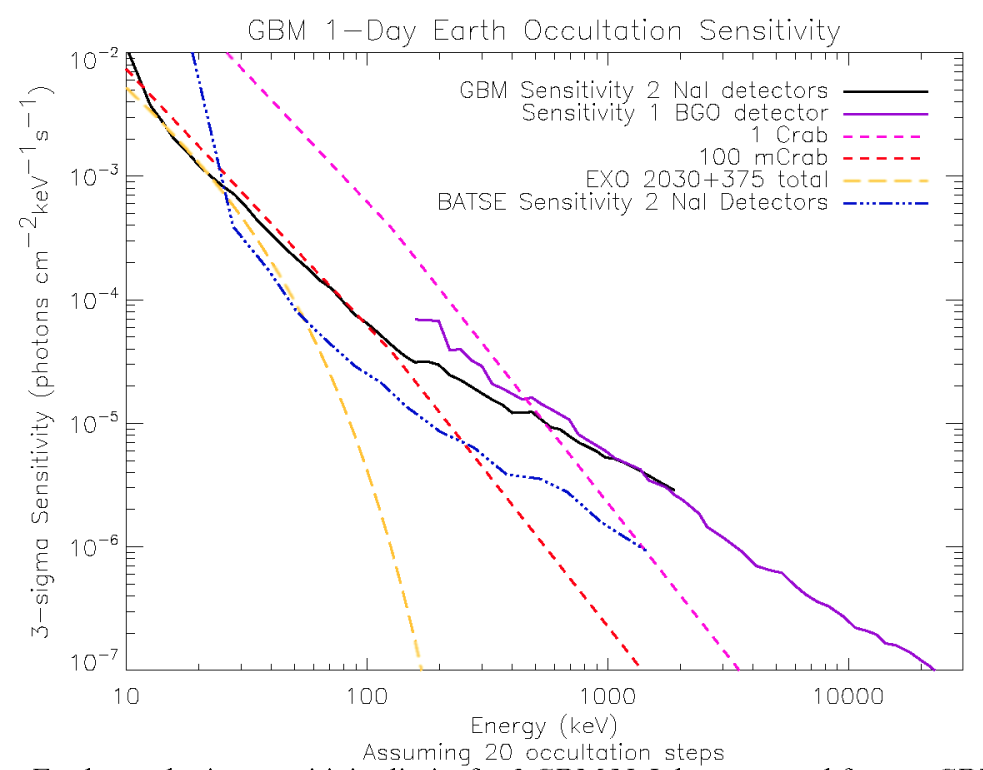

FIGURE 1. The 1-day Earth occultation sensitivity limits for 2 GBM NaI detectors and for one GBM BGO detector for a source at moderate viewing angles. The BATSE sensitivity limit for 2 LADs is shown for comparison. Also shown are the $1 \mathrm{Crab}$ and 100 mCrab spectra, as well as the spectrum of the accreting X-ray pulsar EXO 2030+375. The 1-day sensitivity limit for two GBM NaI detectors below $100 \mathrm{keV}$ is $\sim 90 \mathrm{mCrab}$.

methods developed for BATSE can be readily adapted to the GBM.

Taking into account the expected GBM backgrounds, the GBM Earth occultation sensitivity is estimated to be $\sim 90$ mCrab for $2 \mathrm{NaI}$ detectors in one day (20 occultation steps) below $100 \mathrm{keV}$ for a source at moderate viewing angles (see Figure 1). The GBM will have considerably better sensitivity than BATSE at energies below $\sim 25 \mathrm{keV}$ due to the GBM NaI detectors having only a Be window instead of the plastic scintillator, aluminum honeycomb and aluminum window in front of the BATSE detectors. The GBM sensitivity could be increased by adapting the occultation technique to the use of the Large Area Telescope as an occulter. These occultations could be especially helpful for locating new transients.

The GBM will enable us to continue to monitor known hard $\mathrm{x}$-ray/low-energy gamma-ray transient sources, as well as search for and study new transient objects. When new transients are found, alerts can be provided to other hard $\mathrm{x}$-ray/ gamma-ray instruments such as RXTE, INTEGRAL, and Swift for more sensitive follow-up observations. In addition, studies may be carried out on persistent gamma-ray sources with hard spectra, such as BHCs, AXPs, AGN, etc. Based on the results obtained with BATSE, rich scientific returns can be expected from GBM over the lifetime of the GLAST mission.

\section{REFERENCES}

1. J. C. Ling, et al., ApJS 127, 79 (2000).

2. B. A. Harmon, et al., ApJS 138, 149 (2002).

3. B. A. Harmon, et al., ApJS 154, 585 (2004).

4. G. L. Case, et al., Chinese Journal of Astron. and Astroph. Suppl. 5, 341 (2005).

5. J. C. Ling, and W. A. Wheaton, ApJ 622, 492 (2005a).

6. J. C. Ling, and W. A. Wheaton, Chinese Journal of Astron. and Astroph. Suppl. 5, 80 (2005b).

7. C. A. Meegan, these proceedings (2007). 